\title{
LHCb results on rare leptonic decays of B-mesons
}

\author{
Evgenii Kurbatov, on behalf of the LHCb Collaboration ${ }^{1, *}$ \\ ${ }^{1}$ HSE University, Pokrovskii Bulvar 11, 109028
}

\begin{abstract}
A search for the rare leptonic decays was performed, using the proton-proton collision data collected with the LHCb experiment at center-ofmass energies of $8 \mathrm{TeV}$ and $13 \mathrm{TeV}$. The following analysis are reviewed in this work:
\end{abstract}

- Measurements of the $B_{s}^{0} \rightarrow \mu^{+} \mu^{-}$branching fraction and effective lifetime and search for $B^{0} \rightarrow \mu^{+} \mu^{-}$decays

- Search for the decays $B_{s}^{0} \rightarrow \tau^{+} \tau^{-}$and $B^{0} \rightarrow \tau^{+} \tau^{-}$

- Search for the lepton-flavour violating decays $B_{(s)}^{0} \rightarrow e^{ \pm} \mu^{\mp}$

- Search for the lepton-flavour-violating decays $B_{s}^{0} \rightarrow \tau^{ \pm} \mu^{\mp}$ and $B^{0} \rightarrow \tau^{ \pm} \mu^{\mp}$

- Search for the rare decay $B^{+} \rightarrow \mu^{+} \mu^{-} \mu^{+} v_{\mu}$

All results are consistent with the Standard Model. Nearly all results presented are either unique or the most accurate for the time.

\section{Introduction}

Processes where B meson decays into several leptons in the final state are very powerful sources of new knowledge due to it sensitivity to potential contributions from new mediators and other new physics beyond the Standartd Model (SM). The key features of the decays are:

- Their time-integrated branching fractions are predicted in the SM with small uncertainty due to absence of hadrons in final state

- The decays are sensitive probes for physics beyond the SM

- Within the Standard Model (SM) of particle physics, fully leptonic decays of B mesons are very rare

The search for the rare leptonic decays of B-mesons was performed on the data obtained on $\mathrm{LHCb}$ detector. The $\mathrm{LHCb}$ detector is a single-arm forward spectrometer covering the pseudorapidity range $2<\eta<5$, designed for the study of particles containing b or c quarks. The tracking system provides a measurement of the momentum of charged particles with a relative uncertainty that varies from $0.5 \%$ at low momentum to $1.0 \%$ at $200 \mathrm{GeV} / \mathrm{c}$. Different types of charged hadrons are distinguished using information from two ring-imaging Cherenkov detectors. Photons, electrons and hadrons are identified by a calorimeter system consisting of scintillating-pad and preshower detectors, an electromagnetic and a hadronic calorimeter. Muons are identified by a system composed of alternating layers of iron and multiwire proportional chambers[6][7].

\footnotetext{
*e-mail: evgenii.kurbatov@cern.ch
} 
LHCb data can be divided into 2 different parts, corresponding to the LHC Run1 and Run2, see figure 1. Those parts have a different kinematics and usually can not be processed in exactly the same way. So it's a common situation, when only part of the data is processed in the analysis.

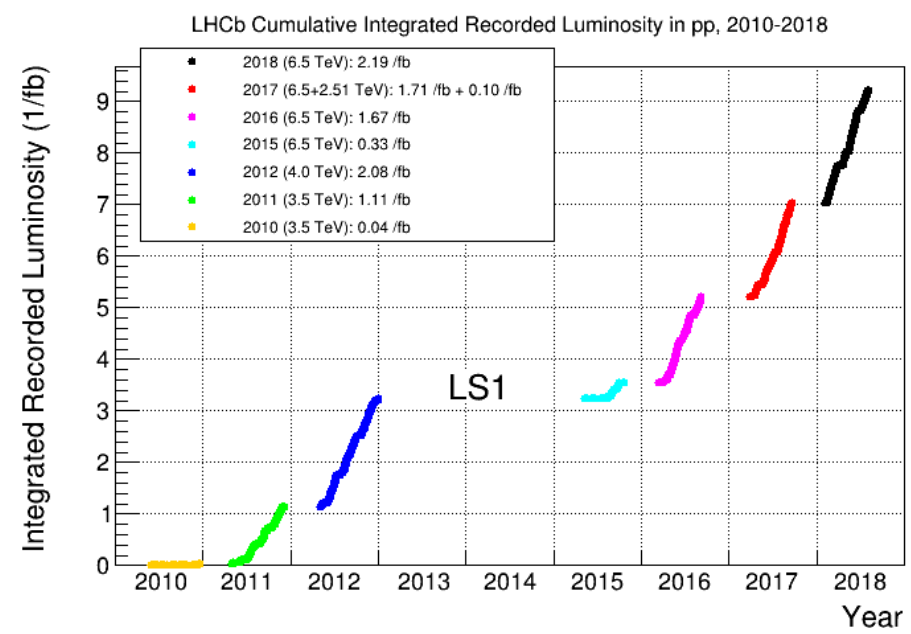

Figure 1. LHCb Cumulative Integrated Recorded Luminosity in pp, 2010-2018

\section{$2 \mathrm{LHCb}$ results}

\subsection{Measurements of the $B_{s}^{0} \rightarrow \mu^{+} \mu^{-}$branching fraction and effective lifetime and search for $B^{0} \rightarrow \mu^{+} \mu^{-}$decays}

Results are based on data collected with the $\mathrm{LHCb}$ detector, corresponding to an integrated luminosity of $1 \mathrm{fb}^{-1}$ of pp collisions at a centre-of-mass energy $\sqrt{\mathrm{s}}=7 \mathrm{TeV}, 2 \mathrm{fb}^{-1}$ at $\sqrt{s}=8 \mathrm{TeV}$ and $1.4 \mathrm{fb}^{-1}$ recorded at $\sqrt{s}=13 \mathrm{TeV}[1]$.

Normalization channels have been used to calculate the branching fractions: $B^{+} \rightarrow$ $J / \psi K^{+}$and $B^{0} \rightarrow K^{+} \pi^{-}$.

$$
\mathcal{B}\left(B_{(s)}^{0} \rightarrow \mu^{+} \mu^{-}\right)=\frac{\mathcal{B}_{\text {norm }} \epsilon_{\text {norm }} f_{\text {norm }}}{N_{\text {norm }} \epsilon_{\text {sig }} f_{d(s)}} \times N_{B_{(s)}^{0} \rightarrow \mu^{+} \mu^{-}} \equiv \equiv \alpha_{B_{(s)}^{0} \rightarrow \mu^{+} \mu^{-}}^{\text {norm }} \times N_{B_{(s)}^{0} \rightarrow \mu^{+} \mu^{-}}
$$

$B_{s}^{0} \rightarrow \mu^{+} \mu^{-}$signal is seen with a significance of 7.8 standard deviations, see figure 3 . The $B_{s}^{0} \rightarrow \mu^{+} \mu^{-}$branching fraction is measured to be $\left(3.0 \pm 0.6_{-0.2}^{+0.3}\right) \times 10^{-9}$, where the first uncertainty is statistical and the second systematic [1]. The SM prediction for this decay is $\mathcal{B}\left(B_{s}^{0} \rightarrow \mu^{+} \mu^{-}\right)=(3.65 \pm 0.23) \times 10^{-9}$ [8]. The previous results from LHCb + CMS data analysis were $\mathcal{B}\left(B_{s}^{0} \rightarrow \mu^{+} \mu^{-}\right)=2.8_{-0.6}^{+0.7} \times 10^{-9}$ [9]. $B_{s}^{0} \rightarrow \mu^{+} \mu^{-}$effective lifetime $\tau\left(B_{s}^{0} \rightarrow\right.$ $\mu^{+} \mu^{-}$) were measured to be $2.04 \pm 0.44 \pm 0.05 \mathrm{ps}$, where the first uncertainty is statistical and the second systematic, see figure 2[1]. No evidence for a $B^{0} \rightarrow \mu^{+} \mu^{-}$signal is found, $\mathcal{B}\left(B^{0} \rightarrow \mu^{+} \mu^{-}\right)<3.4 \times 10^{-10}$ at $95 \%$ confidence. Previous results from LHCb + CMS data analysis are $\mathcal{B}\left(\left(B^{0} \rightarrow \mu^{+} \mu^{-}\right)=3.9_{-1.4}^{+1.6} \times 10^{-10}\right.$ [9].

All results are in agreement with the Standard Model expectations 


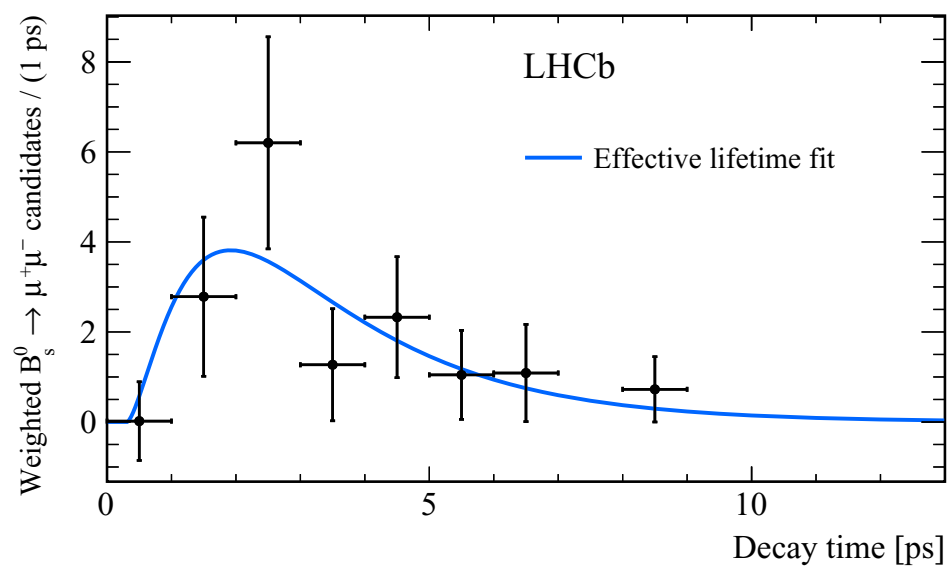

Figure 2. Effective lifetime fit for the $B_{s}^{0} \rightarrow \mu^{+} \mu^{-}$decay[1]

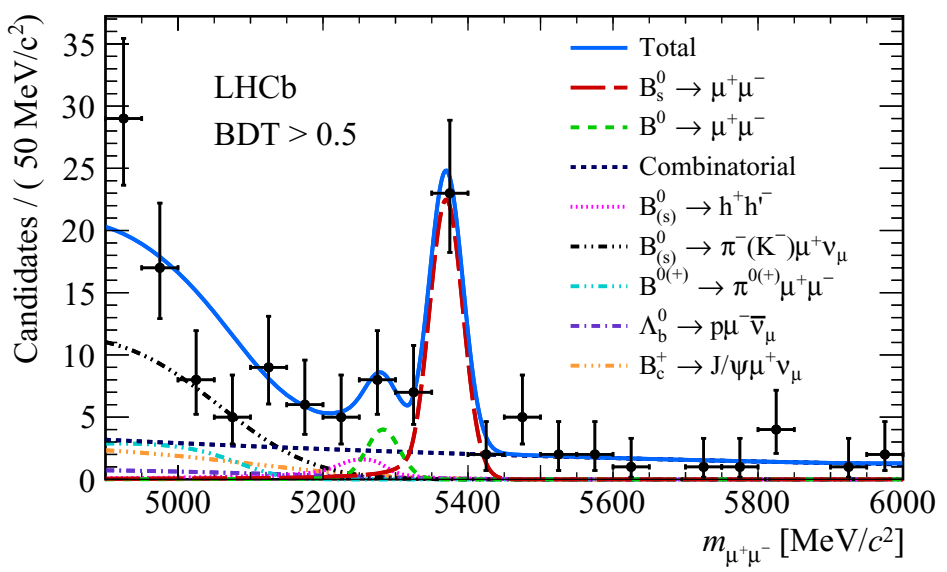

Figure 3. Mass distribution of the selected $B_{s}^{0} \rightarrow \mu^{+} \mu^{-}$candidates (black dots) with BDT $>0.5$. The result of the fit is overlaid, and the different components are detailed[1].

\subsection{Search for the decays $B_{s}^{0} \rightarrow \tau^{+} \tau^{-}$and $B^{0} \rightarrow \tau^{+} \tau^{-}$}

The analysis is performed with proton-proton collision data corresponding to integrated luminosities of $1.0 \mathrm{fb}^{-1}$ and $2.0 \mathrm{fb}^{-1}$ recorded with the LHCb detector at centre-of-mass energies of 7 and $8 \mathrm{TeV}$, respectively. The $\tau$ leptons are reconstructed through the decay $\tau \rightarrow \pi^{-} \pi^{+} \pi^{-} \nu_{\tau}[2] . B_{s}^{0}$ and $B^{0}$ mesons can not be discerned due to the $v$ in the final state, which can not be detected by the LHCb detector. Analysis strategy is built on segregating events into 3 different groups according to the pions position on the figure 4 .

Assuming no contribution from $B^{0} \rightarrow \tau^{+} \tau^{-}, \mathcal{B}\left(B_{s}^{0} \rightarrow \tau^{+} \tau^{-}\right)<6.8 \times 10^{-3}$ at 95\% CL. And in reverse, if no contribution from $B_{s}^{0} \rightarrow \tau^{+} \tau^{-}$is assumed, $\mathcal{B}\left(B^{0} \rightarrow \tau^{+} \tau^{-}\right)<2.1 \times 10^{-3}$ at $95 \% \mathrm{CL}$. Previous limit (BaBar collaboration) is $\mathcal{B}\left(B^{0} \rightarrow \tau^{+} \tau^{-}\right)<4.10 \times 10^{-3}$ at $90 \%$ 


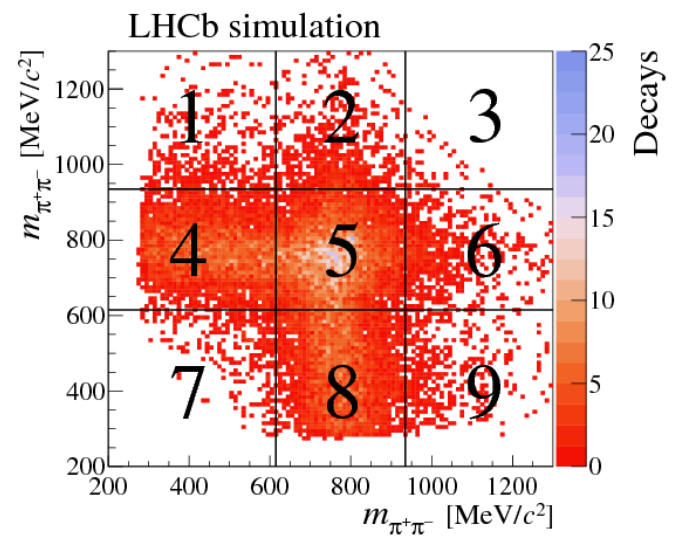

Figure 4. Two-dimensional distribution of the invariant masses $m_{\pi^{+} \pi^{-}}$of the two oppositely charged two-pion combinations for simulated $B_{s}^{0} \rightarrow \tau^{+} \tau^{-}$candidates. The distribution is symmetric by construction. The vertical and horizontal lines illustrate the sector boundaries[2].

CL[10]. These results correspond to the first direct limit on $\mathcal{B}\left(B_{s}^{0} \rightarrow \tau^{+} \tau^{-}\right)$and the world's best limit on $\mathcal{B}\left(B^{0} \rightarrow \tau^{+} \tau^{-}\right)$.

\subsection{Search for the lepton-flavour violating decays $B_{(s)}^{0} \rightarrow e^{ \pm} \mu^{\mp}$}

A search for the lepton-flavour violating (LFV) decays $B_{(s)}^{0} \rightarrow e^{ \pm} \mu^{\mp}$ was performed using pp collision data collected at centre-of-mass energies of 7 and $8 \mathrm{TeV}$, corresponding to a total integrated luminosity of $3 \mathrm{fb}^{-1}$ [3]. Two normalisation channels were used: the $B^{0} \rightarrow K^{+} \pi^{-}$ decay which has a similar topology to that of the signal, and the $B^{+} \rightarrow J / \Psi K^{+}$decay, with $J / \Psi \rightarrow \mu^{+} \mu^{-}$, which has an abundant yield and a similar purity and trigger selection[3].

The result fit was performed independently for the different BDT bins. Also 2 different approaches were taken into account: the direct one and the one where the momentum of the final state particles was corrected using the information about bremsstrahlung photons.

No excesses are observed and upper limits on the branching fractions are set to $\mathcal{B}\left(B_{s}^{0} \rightarrow\right.$ $\left.e^{ \pm} \mu^{\mp}\right)<6.3 \times 10^{-9}$ and $\mathcal{B}\left(B^{0} \rightarrow e^{ \pm} \mu^{\mp}\right)<1.3 \times 10^{-9}$ at $95 \%$ CL [3]. These results represent the best upper limits to date and are a factor 2 to 3 better than the previous results from $\mathrm{LHCb}$ $\left(\mathcal{B}\left(B_{s}^{0} \rightarrow e^{ \pm} \mu^{\mp}\right)<1.4 \times 10^{-8}\right.$ and $\mathcal{B}\left(B^{0} \rightarrow e^{ \pm} \mu^{\mp}\right)<3.7 \times 10^{-9}$ at $95 \%$ CL) [11].

\subsection{Search for the lepton-flavour-violating decays $B_{s}^{0} \rightarrow \tau^{ \pm} \mu^{\mp}$ and $B^{0} \rightarrow \tau^{ \pm} \mu^{\mp}$}

A search for $B_{s}^{0} \rightarrow \tau^{ \pm} \mu^{\mp}$ and $B^{0} \rightarrow \tau^{ \pm} \mu^{\mp}$ decays is performed using data corresponding to an integrated luminosity of $3 \mathrm{fb}^{-1}$ of proton-proton collisions, recorded with the LHCb detector in 2011 and 2012 [4]. The $\tau$ lepton is reconstructed in the $\tau \rightarrow \pi^{-} \pi^{+} \pi^{-} v_{\tau}$ channel. Again, $B_{s}^{0}$ and $B^{0}$ mesons can not be discerned due to the $v$ in the final state, which can not be detected by the $\mathrm{LHCb}$ detector. 

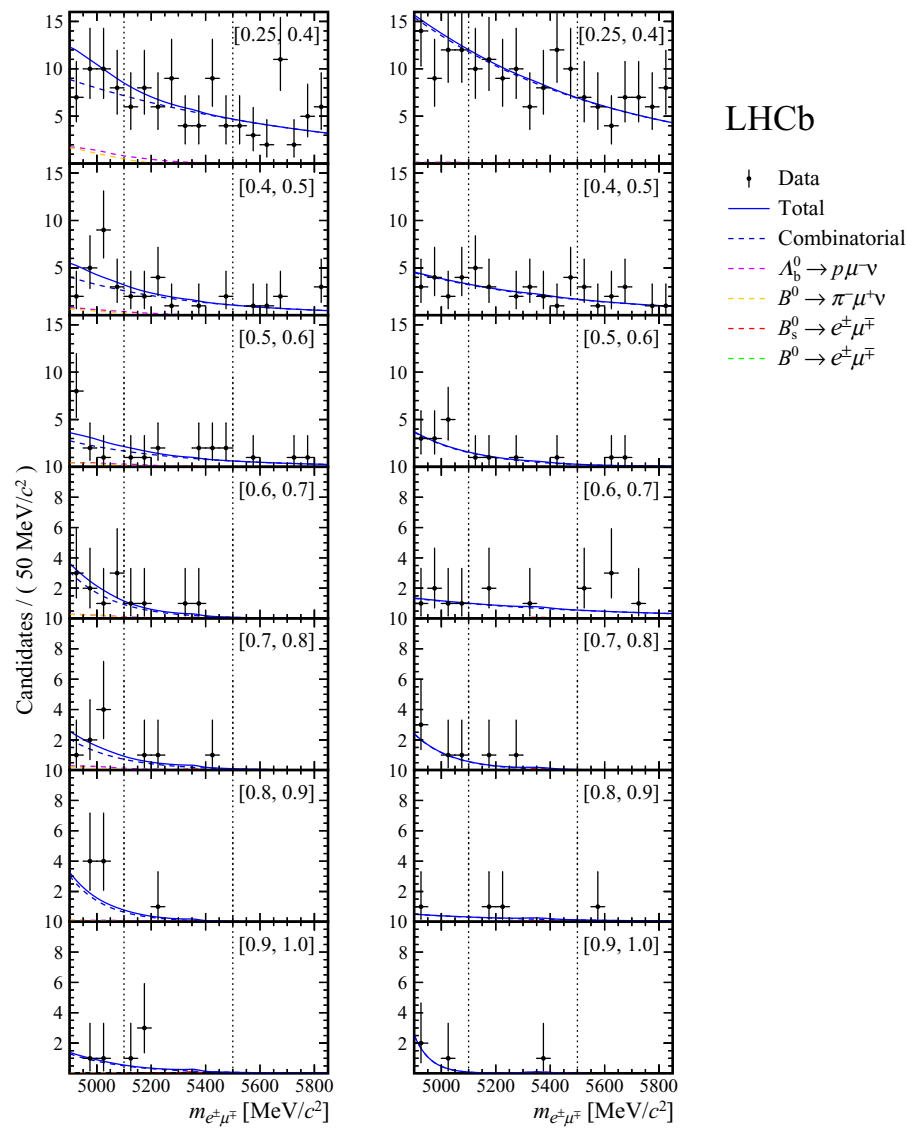

Figure 5. Distributions of the invariant mass of the $B_{(s)}^{0} \rightarrow e^{ \pm} \mu^{\mp}$ candidates, $m_{e^{ \pm} \mu^{\mp}}$, divided into bins of BDT response and two bremsstrahlung categories (left) without and (right) with bremsstrahlung photons recovered. The result of the fit is overlaid and the different components are detailed. The edges of the range that was examined only after finalising the selection and fit procedure are delimited by gray dashed vertical lines. This region includes $90 \%$ of the potential signal candidates. Given the result obtained from the fit, the $B^{0} \rightarrow e^{ \pm} \mu^{\mp}$ component is not visible in the plots[3].

$\mathcal{B}\left(B_{s}^{0} \rightarrow \tau^{ \pm} \mu^{ \pm}\right)<4.2 \times 10^{-5}$ at $95 \%$ CL (Assuming no contribution from $B^{0} \rightarrow \tau^{ \pm} \mu^{ \pm}$). $\mathcal{B}\left(B^{0} \rightarrow \tau^{ \pm} \mu^{ \pm}\right)<1.4 \times 10^{-5}$ at $95 \%$ CL (Assuming no contribution from $B_{s}^{0} \rightarrow \tau^{ \pm} \mu^{ \pm}$) [4]. These are the first limit on $\mathcal{B}\left(B_{s}^{0} \rightarrow \tau^{ \pm} \mu^{ \pm}\right)$and the world's best limit on $\mathcal{B}\left(B^{0} \rightarrow \tau^{ \pm} \mu^{ \pm}\right)$.

\subsection{Search for the rare decay $B^{+} \rightarrow \mu^{+} \mu^{-} \mu^{+} v_{\mu}$}

A search for the rare leptonic decay $B^{+} \rightarrow \mu^{+} \mu^{-} \mu^{+} v_{\mu}$ is performed using proton-proton collision data corresponding to an integrated luminosity of $4.7 \mathrm{fb}^{-1}$ collected by the LHCb experiment. The search is carried out in the region where the lowest of the two $\mu^{+} \mu^{-}$mass combinations is below $980 \mathrm{MeV} / \mathrm{c}^{2}$. The branching fraction of a $B^{+} \rightarrow \mu^{+} \mu^{-} \mu^{+} v_{\mu}$ signal is obtained by normalising to the $B^{+} \rightarrow J / \Psi\left(\rightarrow \mu^{+} \mu^{-}\right) K^{+}$decays [5].

No signal is observed for the $B^{+} \rightarrow \mu^{+} \mu^{-} \mu^{+} v_{\mu}$ decay, see figure 7. An upper limit of $1.6 \times 10^{-8}$ at $95 \%$ confidence level is set on the branching fraction, where the lowest of the two 

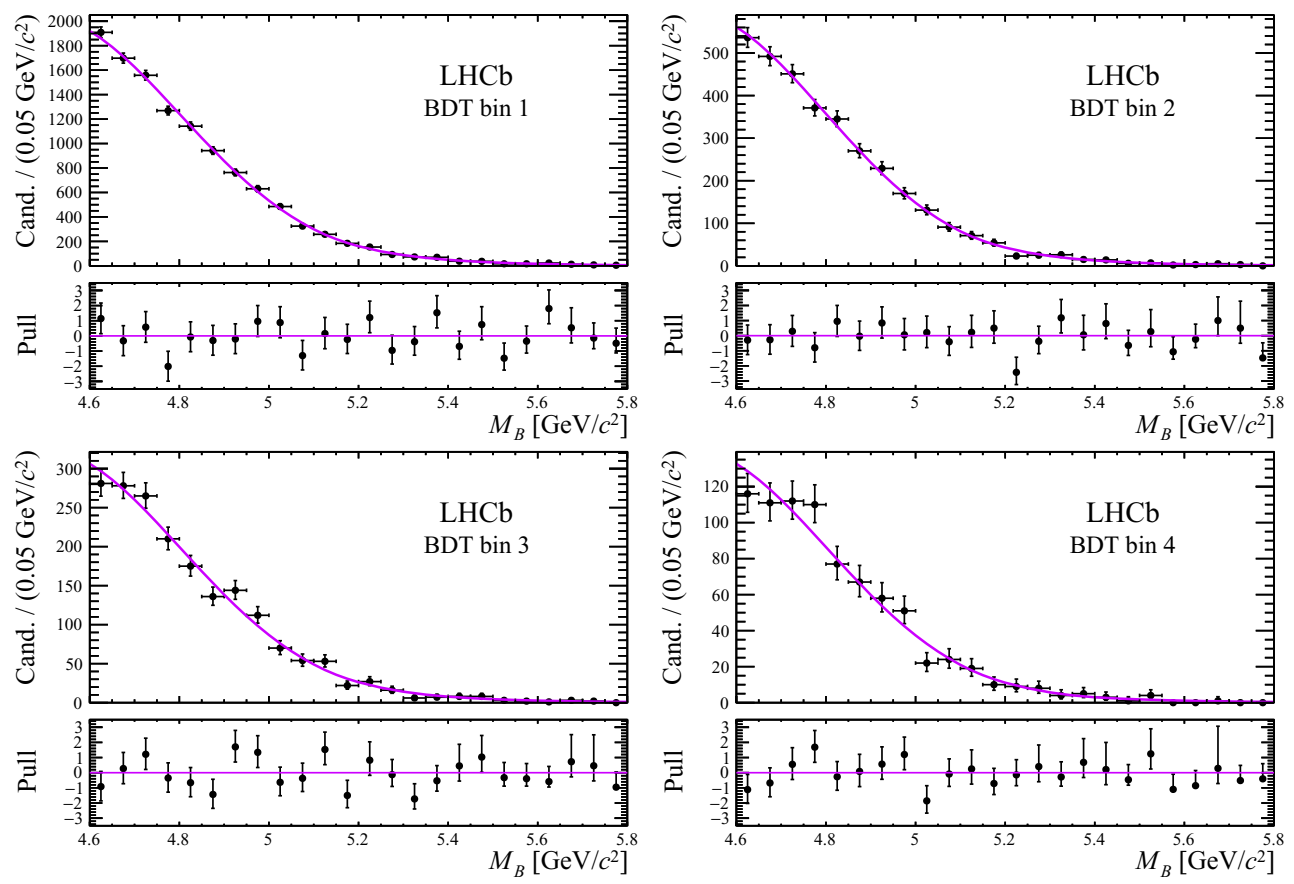

Figure 6. Distributions of the reconstructed B invariant-mass in data in the four final BDT bins with the projections of the fit for the $B_{s}^{0}$ signal-only hypothesis overlaid. The lower-part of each figure shows the normalised residuals [4].

$\mu^{+} \mu^{-}$mass combinations is below $980 \mathrm{MeV} / \mathrm{c}^{2}$. The limit for the full kinematic region stays the same under the assumption that the decay is dominated by intermediate vector mesons $[5]$.

\section{Conclusions}

Lot of rare B mesons decays were studied by the LHCb team. All results are consistent with the Standard Model. Nearly all results presented are either unique or the most accurate for the time. 


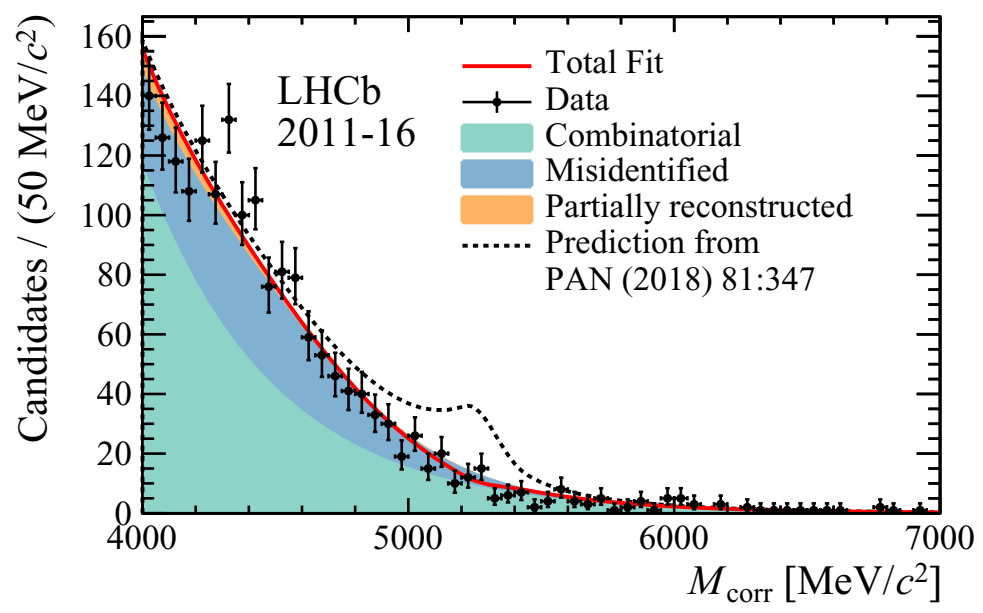

Figure 7. Fit to the mass distribution of the selected $B^{+} \rightarrow J / \Psi K^{+}$candidates. The combinatorial background (purple) and misidentified $B^{+} \rightarrow J / \Psi \pi^{+}$decays (orange) are stacked up while the $B^{+} \rightarrow$ $J / \Psi K^{+}$signal is shown as a dashed line. The data points are shown as black points with the total fit overlaid as a red solid line [5]. 


\section{References}

[1] R. Aaij et al. (LHCb Collaboration), Phys. Rev. Lett., 118, 191801 (2017)

[2] R. Aaij et al. (LHCb Collaboration), Phys. Rev. Lett., 118, 251802 (2017)

[3] R. Aaij et al. (LHCb Collaboration), JHEP 1803, 078 (2018)

[4] R. Aaij et al. (LHCb Collaboration), arXiv:1905.06614 [hep-ex]

[5] R. Aaij et al. (LHCb Collaboration), Eur. Phys. J. C 79, 675 (2019)

[6] LHCb collaboration, A. A. Alves Jr. et al., JINST 3, S08005 (2008)

[7] LHCb collaboration, R. Aaij et al., Int. J. Mod. Phys. A30, 1530022 (2015)

[8] Christoph Bobeth, Martin Gorbahn et al., Phys. Rev. Lett. 112, 101801 (2014)

[9] CMS Collaboration \& LHCb Collaboration, Nature 522, 68-72 (2015)

[10] B. Aubert et al. (BABAR Collaboration), Phys. Rev. Lett. 96, 241802 (2006)

[11] R. Aaij et al. (LHCb Collaboration), Phys. Rev. Lett. 111, 141801 (2013) 